\title{
Application of a dermatopharmacokinetic (DPK) method for bioequivalence assessment of topical metronidazole creams
}

\author{
Seeprarani Rath ${ }^{1}$, Ashmita Ramanah $^{1}$, Charles Bon ${ }^{2}$, Isadore Kanfer ${ }^{1,3}$ \\ ${ }^{1}$ Biopharmaceutics Research Institute, Rhodes University, Grahamstown, South Africa; ${ }^{2}$ Biostudy Solutions LLC., Wilmington, NC, \\ USA; ${ }^{3}$ Leslie Dan College of Pharmacy, University of Toronto, Toronto, ON, Canada
}

Corresponding Author: Isadore Kanfer, Leslie Dan Faculty of Pharmacy, University of Toronto

Toronto, ON, Canada M5S 3M2,; Tel: +1-416-568-0322; e-mail: izzy.kanfer@utoronto.ca

Received, October 22, 2020; Revised, November 2, 2020; Accepted, November 3, 2020; Published, November 6, 2020

\begin{abstract}
Purpose: The main aim of the current research was to develop and apply a dermatopharmacokinetic (DPK) approach for the bioequivalence assessment of metronidazole (MTZ) topical cream products, indicated in the treatment of rosacea. Methods: A DPK methodology using tape stripping (TS) technique was developed by investigating the factors that may influence the TS results viz. tapes, dose durations, number of tapes to be used, pressure application, dose applied and gravimetric analysis of the tapes. An initial dose duration study was performed on 6 healthy participants to determine an appropriate application time duration using the $E_{\max }$ model. The stratum corneum (SC) thickness was normalised between participants using transepidermal water loss (TEWL) measurements. A pivotal study was conducted using both the arms of 10 healthy human participants to demonstrate the ability of the TS method for bioequivalence assessment by comparing the reference product, Metrocreme ${ }^{\circledR}(0.75 \% \mathrm{MTZ})$, to itself as a positive control and including products with higher and lower strengths of MTZ to serve as negative controls in order to confirm bioinequivalence. Results: Whereas the reference was found to be bioequivalent when compared to itself, the creams containing $0.56 \%$ and $0.95 \% \mathrm{MTZ}$ (negative controls) were not bioequivalent (bioinequivalent). Furthermore, another product containing $0.75 \% \mathrm{MTZ}$ was also assessed and was found to be bioequivalent to the reference product. In addition, the use of both forearms of each participant offered an important advantage of significantly reducing the number of human subjects required to demonstrate $\mathrm{BE}$ with a high statistical power of $>80 \%$. Conclusion: The data obtained provides compelling evidence that the developed TS method has the potential to be a cost-effective surrogate alternative for lengthy and expensive clinical trials. Consequently, its application can facilitate faster development of generic products which would, in turn, lower the economic burden of healthcare.
\end{abstract}

\section{INTRODUCTION}

The cost of prescription medicines is an ongoing concern leading to unaffordability and, consequently, patients often defer or even abandon treatment. The availability of generic products provide lower-priced competing versions of medications and therefore facilitate patient access to marketed medicines $(1,2)$. However, demonstration of bioavailability/ bioequivalence (BA/BE) of topical semi-solid dosage forms intended for local action has been a major challenge amongst scientific researchers and regulatory agencies around the world (3). Whereas the determination of drug concentrations in blood following extravascular administration of drug products intended for systemic circulation has been successfully used to determine $\mathrm{BA} / \mathrm{BE}$, such an approach is not valid for the $\mathrm{BE}$ assessment of topically applied products for local action. Apart from the FDA's vasoconstriction assay (VCA) (4) which provides only for the $\mathrm{BE}$ testing of topical corticosteroid products, and more recently, several product-specific guidances intended for biowaiver applications (5-10), clinical trials have been the only means whereby a generic company can demonstrate $\mathrm{BA} / \mathrm{BE}$ of other topical dosage forms intended for local activity (3).

For extravascularly administered drug products intended for systemic absorption, the area under the curve $(A U C)$ obtained from plasma drug concentration $v s$ time profiles gives an estimate of their BAs (11). This, however, is not applicable to topical products intended for local action since the site of application is also the intended site of action with minimal systemic uptake (12). Additionally, since the dose applied is generally low $\left(\sim 5 \mathrm{mg} / \mathrm{cm}^{2}\right.$ of the product), the determination of the kinetics of uptake and elimination following topical administration is impractical $(13,14)$. Stratum 
corneum (SC) functions as a rate limiting barrier for topically applied drug products and is also the site of action for a number of topically administered drug products, such as antifungal agents, while some others like MTZ, act in the lower layers of the epidermis or the dermis. Tape stripping (TS) enables the quantitative determination of drug amounts in stripped SC. The dermatopharmacokinetic (DPK) approach using TS is the most commonly used and accepted method for SC sampling because it offers a relatively cheap, simple and quick method to do so (15). Moreover, it is relatively non-invasive and does not influence inflammatory components (16).

The utility of TS to determine BA/ BE of topical products led to the publication of a Guidance for Industry entitled “Topical Dermatological Drug Product NDAs and ANDAs - In Vivo Bioavailability, Bioequivalence, In Vitro Release, and Associated Studies" in 1998 (15). Although the protocol described in the guidance yielded promising outcomes, the FDA withdrew the guidance in 2002 (17) following major concerns regarding the appropriateness of the DPK method to assess topical products whose site of action was not the SC and particularly its lack of reproducibility between different laboratories. The basis of the latter concern was the contradictory $\mathrm{BE}$ assessment results for tretinoin gel products obtained by two reputed independent laboratories. The reasons for the variabilities were attributed to the lateral spreading of the formulations and differences in the TS protocols used between laboratories $(18,19)$.

The high degree of variability associated with the FDA's DPK protocol necessitated recruitment of a large number of participants to achieve statistical power of $>80 \%$ to obtain satisfactory results $(20,21)$. This variability may be attributed to the inconsistencies in the amount of SC stripped with each tape strip, the amount of drug present in the discarded tape strips and inefficiency of the residual product removal procedure (20). Some of the additional limitations of the original DPK guidance include the trial and error approach employed to determine the time points for TS, the length of time and the amount of manpower required for the procedure (22). The guidance also did not take into account the inter individual differences in the SC characteristics, especially the thickness $(18,20)$.

After the withdrawal of the guidance in 2002, various attempts $(18,20,23-25)$ have been made to develop a standardised TS method and to minimise the variability associated with it. In 2003,
Japan's National Institute of Health Sciences published a guideline that made provisions to include TS for BE studies of generic products for topical use (26). Other countries that accepted TS for BE assessment of topical products included South Africa (27) and Brazil (28) until recently when the European Medicines Agency (EMA) published a draft guideline on quality and equivalence of topical products in 2018 (29). More recently, a new FDA draft guidance has been published, whose objective is to facilitate the development and approval of generic products (30).

The use of the FDA's 8 time point profile to determine the $A U C$ has largely been criticized due to the associated impracticalities (18). An alternative approach (31) involving the comparison of the total amount of drug found in the $\mathrm{SC}$ has been used instead of using AUCs to compare cream and ointment formulations using a single time point. However, this approach involves a relatively rugged procedure and assumes that the amount of SC is the same amongst the study participants. The results also largely depend upon the amount of dose applied and the chosen dose duration (DD). Some other studies $(20,32)$ have been conducted by employing two time points - representing the "uptake" and "clearance" phases.

Several studies have demonstrated the utility of TS to establish bioequivalence between products $(18,21,33-38)$. N'Dri-Stempfer et al. (18) identified the sources of variabilities in the DPK approach and designed an improved method that minimised the influence of these variables to assess bioequivalence between topical products containing $1 \%$ econazole nitrate. The inclusion of a negative control i.e. non-bioequivalent product was suggested in order to avoid misclassification of products. Ortiz et al. (34) developed a TS method that had the necessary properties to detect differences in drug amounts and establish bioequivalence. Similarly, Parfitt et al. (24) demonstrated the potential of TS to establish bioequivalence between topical products containing clotrimazole using an approach wherein the skin thickness between the participants was normalised and the tape strips were individually weighed, extracted and analysed. In a more recent study, Nallagundla et al. (23) developed and optimised a TS methodology and used it to accurately assess bioequivalence between topical products containing acyclovir with a high statistical power $(>90 \%)$. Importantly, these methodologies avoided numerous measurements of drug amount by performing TS only once, at a DD within the uptake 
phase, since this phase is sensitive to formulation performance, the key component in bioequivalence testing.

The PK parameters, area under the curve $(A U C)$, peak plasma drug concentration $\left(C_{\max }\right)$ and/or maximum drug quantity per unit area $\left(\mathrm{ng} / \mathrm{cm}^{2}\right)$ in the SC $\left(Q_{\max }\right)$ and thermodynamic parameters, SC-vehicle partition coefficient of the drug and diffusivity $(D)$ of the drug across the SC of thickness $(L)$ i.e. $D / L^{2}$ have been employed for topical BA and BE assessment (21,24,39-41) whereas recently, Ozdin et al. (42) proposed a refined strategy that eliminated some of the limitations and complexities in DPK approaches used thus far using the TS procedure described by Nallagundla et al. (23). A total of 56 samples per subject were collected for analysis. This number would have increased if the FDA proposed method $(14 \mathrm{TS} \times 8$ sites for Test and $14 \mathrm{TS} \times 8$ sites for Ref $=224)$ or the Two-Time method (28 TS for Test and $28 \mathrm{TS}$ for Reference in each of uptake and clearance phases $=112$ ) were used. As a result, a DPK profile that consisted of the SC drug quantity $(\mu \mathrm{g})$ as a function of time was generated using a novel method by attributing one time point to each stripped layer (i.e. DD of $8 \mathrm{~min}+$ each TS procedure lasting $30 \mathrm{~s}$ ). This approach obviates the need to tape strip at many DDs and simplifies the DPK procedure by eliminating the need for gravimetric analysis of the tape strips. In view of the fact that the percentage of the dose absorbed after topical application is very small and since topical dermatological products are not intended to deliver the drug into the systemic circulation, PK parameters such as clearance and volume of distribution are not relevant using this method (42).

The choice of DD has generally been unsubstantiated and usually the dose is left on for an arbitrary period of time. Since sampling at a time when the amount of drug in the SC has reached steady state is likely to mask differences in formulations, it is necessary to have a validated method to ensure that the chosen dose duration lies on a sensitive part of the dose-response curve (dose duration $v s A U C$ ). It is important to ensure that the pivotal study is carried out at a dose duration which can provide the necessary discriminatory power to identify significant differences between products. Hence, the approach described in the FDA's VCA guidance (4) was employed to determine the dose duration for topical MTZ creams.

From the literature accessed, the need to develop a standardised TS method with necessary discriminatory capability to accurately determine bioequivalence was realized. Hence, positive and negative controls were included. In addition, unlike some previous studies, where only one arm of each participant was used, the current study describes the use of both arms in the same participant in order to reduce the number of participants required to show $\mathrm{BE}$ using TS.

\section{METHODS}

\section{Chemicals}

MTZ was obtained from Sigma-Aldrich and stored in a cool, dry area that was free from light exposure. HPLC-grade methanol (200 UV ROMIL-SpSTM Super Purity Solvent) was obtained from Romil Ltd. (Waterbeach, Cambridge, UK). The water used for chromatography was prepared by reverse osmosis, followed by filtration through a Milli-Q system (Millipore, Bedford, MA, USA).

\section{Formulations}

Metrocreme $^{\circledR}$ (Galderma Laboratorium GmbH, Düsseldorf, Germany) containing $0.75 \%$ MTZ was used as the reference MTZ cream and specially manufactured creams containing equal strength $(0.75 \%), 25 \%$ less $(0.56 \%)$ and $26 \%$ more $(0.95 \%)$ MTZ than the reference product were used as test products, $T_{1}, T_{2}$ and $T_{3}$, respectively to establish the utility of the developed TS method for the assessment of bioequivalence. A placebo cream was used for ultra-performance liquid chromatography (UPLC) method validation.

\section{Equipment}

A calibrated MX5 Mettler $^{\circledR}$ Toledo Microbalance (Mettler ${ }^{\circledR}$ Inc, Zurich, Switzerland) that is sensitive enough to measure low masses was used for weighing standards and tape strips. Furthermore, a static eliminator (AD 1683 DC, A\&D Company Ltd., Tokyo, Japan) was used to neutralize the static build up on the tape strips to ensure the accuracy and reliability of the masses obtained. Micropipettes P100 and P1000 (PipetmanTM, Gilson ${ }^{\circledR}$, Villiersle-Bel, France) were used to transfer standard solutions and tape strip extracts.

The amounts of MTZ in the tape strips were determined using a Waters Acquity UPLC system equipped with a photo diode array detector (PDA), Empower $^{\circledR} 3$ data acquisition system (Waters, Milford, MA, USA). The chromatographic separation was achieved by using an Acquity UPLC BEH C18 $1.7 \mu \mathrm{m}(2.1 \times 100 \mathrm{~mm})$. A mobile phase of methanol/water $(40 / 60 \mathrm{v} / \mathrm{v})$ at a flow rate of 0.2 $\mathrm{mL} / \mathrm{min}$ was used, and the eluate was monitored at 
a wavelength of $318 \mathrm{~nm}$. Samples $(2 \mu \mathrm{L})$ were injected at ambient temperature during analysis.

\section{Tape selection}

Five types of adhesive tapes viz. Micropore ${ }^{\mathrm{TM}}$ Surgical Tape (3M, Johannesburg, South Africa), Transpore $^{\mathrm{TM}}$ Surgical Tape (3M, Johannesburg, South Africa), Scotch ${ }^{\mathrm{TM}}$ Magic Tape (3M, Johannesburg, South Africa), Sellotape ${ }^{\circledR}$ Crystal Clear Tape (SSC, Cleveland, South Africa) and Sellotape ${ }^{\circledR}$ Easi Tear Invisi Tape (SSC, Cleveland, South Africa) were investigated as potential candidates. Scotch ${ }^{\mathrm{TM}}$ Magic Tape was chosen due to its high extraction efficiency, good adhesiveness, absence of interference during analysis, and ease of handling.

\section{UPLC method validation}

The chosen tapes were individually cut into squares $(2.4 \times 2.4 \mathrm{~cm})$, spiked with $10 \mu \mathrm{L}$ of the relevant standard solutions ranging from $5-500 \mu \mathrm{g} / \mathrm{mL}$ methanolic solution of MTZ that would yield samples with concentrations of $0.05-5 \mu \mathrm{g} / \mathrm{mL}$ upon extraction. Water $(1 \mathrm{~mL})$ was added to the tubes and the samples were subjected to ultrasonic treatment for $20 \mathrm{~min}$ at $55^{\circ} \mathrm{C}$ using an ultrasonic water bath (model 702, Scientech Ultrasonicator, United Scientific (Pty) Ltd, Port Elizabeth, South Africa). The samples were then vortexed for $4 \mathrm{~min}$ on an Eppendorf MixMateTM (model PCB-08, Eppendorf, Hamburg, Germany) at $1800 \mathrm{rpm}$, followed by centrifugation for $10 \mathrm{~min}$ (Eppendorf Centrifuge 5415, Hamburg, Germany) at $1300 \mathrm{rpm}$ and filtered through a Durapore ${ }^{\circledR}(\mathrm{PVDF}, 0.22 \mu \mathrm{m}$, $33 \mathrm{~mm}$, Merck Millipore Ltd., Ireland) syringe filter prior to chromatographic analysis. Additionally, unspiked tapes were extracted and used as blanks. The UPLC method was validated in accordance with the International Council for Harmonization (ICH) guidelines (43). The validation criteria and procedures used to validate them are described below.

\section{Selectivity, specificity}

In order to assess the ability of the method to detect and identify MTZ in the presence of other components of the cream and stripped SC, four control solutions i.e. extracts from unspiked tape (negative control $T C_{n}$ ), tape spiked with MTZ to yield $1 \mu \mathrm{g} / \mathrm{mL}$ extract (positive control $T C_{p}$ ), unspiked tape used to strip the skin following application of placebo (matrix negative control $T C_{n m}$ ) and tape used to strip the skin following application of placebo spiked with MTZ to yield 1 $\mu \mathrm{g} / \mathrm{mL}$ extract (matrix positive control $T C_{p m}$ ). The samples thus obtained were investigated for the presence of interfering peaks due to the components from the placebo cream and $\mathrm{SC}$ and to ensure that the integrity of the MTZ peaks was not compromised following TS studies. According to the acceptance criteria, the difference between the mean retention times of positive control and positive matrix control i.e. $R T_{p}$ and $R T_{p m}$, respectively, should not be more than $10 \%$. Furthermore, the negative controls were checked for the presence of any detectable MTZ peaks at the specified retention time.

\section{Linearity}

A set of eight calibration standards with concentrations of $0.05,0.1,0.2,0.5,1,2,4$, and 5 $\mu \mathrm{g} / \mathrm{mL}$ were analysed in six runs and a linear regression curve established for each of these runs. The predetermined specifications for the validation were that $75 \%$ of the standards, and at least one standard per concentration, should meet the following acceptance criteria: The measured concentration should lie within $\pm 15 \%$ of the nominal concentration $\left(T C_{n o m}\right)$, except for the lowest concentration which should lie within $\pm 20 \%$ of $T C_{n o m}$. Additionally, the coefficient of determination $\left(R^{2}\right)$ values of each regression curve calculated for each batch run should be $>0.95$.

\section{Accuracy and precision}

The tapes from the placebo cream TS studies were spiked with MTZ to obtain concentrations of 0.5 $\left(T S_{0.5}\right), 1\left(T S_{1}\right)$ and $5 \mu \mathrm{g} / \mathrm{mL}\left(T S_{5}\right)$ to be used as accuracy standards. Three runs were conducted on a single day which were used to determine the accuracy and intra-day precision. Additionally, a run was carried out every day for three consecutive days to determine inter-day precision. To demonstrate accuracy the average measurements $\left(T S_{\text {meas }, a c}\right)$ from the runs for intra-day precision and from each of the three solutions $(n=3)$ should not deviate by more than $\pm 15 \%$ for high and medium concentrations and by not more than $\pm 20 \%$ for low concentration from the respective nominal concentrations $\left(T S_{\text {nom }}\right)$. The intra- and inter-day precisions were evaluated by calculating the $\mathrm{CV}$ for each of the three runs carried out on day 1 for intraday precision and across the three days for inter-day precision.

\section{Recovery}

To confirm that most of the drug present in tape strips was extracted, the percent recoveries of the 
samples obtained after extraction were compared to the standards corresponding to their $T C_{n o m}$. The amount of drug recovered after extraction should be greater than $85 \%$ (23).

\section{Robustness}

Different column temperatures $\left(22.5\right.$ and $27.5^{\circ} \mathrm{C}$ instead of $\left.25^{\circ} \mathrm{C}\right)$ and different flow rates $(0.18$ and $0.22 \mathrm{~mL} / \mathrm{min}$ instead of $0.2 \mathrm{~mL} / \mathrm{min}$ ) were used to assess robustness. These were compared with the average measurements from the linearity validation to establish robustness. Additionally, a different column of the same type was used on a different day and validated for its robustness.

\section{Stability}

Solutions $T S_{0.5}, T S_{1}$ and $T S_{5}$ were used to test the short-term stability of the samples on the bench top $\left(21 \pm 1^{\circ} \mathrm{C}\right)$, in the UPLC sample tray $\left(21^{\circ} \mathrm{C}\right)$ and in the refrigerator $\left(4 \pm 1^{\circ} \mathrm{C}\right)$ for 2 days. Sample stability was considered acceptable if the mean measured concentrations of the solutions/calibration standards did not deviate by more than $\pm 15 \%$ from the nominal concentration $\left(T S_{\text {nom }}\right)$ for the solutions with high and medium concentrations and by not more than $\pm 20 \%$ for the lowest concentration.

\section{Lower limit of quantification (LLOQ) and limit of detection (LOD)}

The LLOQ and the LOD were determined by injecting a 0.05 and $0.1 \mu \mathrm{g} / \mathrm{mL}$ solution of MTZ six times.

\section{Participants}

Healthy participants between 18 and 50 years of age who were available for the entire study period and willing to adhere to the protocol requirements and able to give informed consent were included in the current study. Participants with a known allergy/ hypersensitivity to MTZ or significant contact allergy to the adhesive on tape used during the study were excluded from the study. In addition, participants having excessively hirsute ventral forearms and/or abrasions on the application sites or with excessive exposure to direct sunlight or artificial sun-tanning within 1 month of the study date or participated in any other clinical study/trial within 2 months of the dose application were not included in the study. Participants were asked to refrain from applying emollients, skin conditioning creams and/or tanning lotions to their forearms 24 hours before the study and from performing strenuous exercise for a period of 12 hours prior to the study. No prescription or over-the-counter medication (except oral contraceptive pills) was allowed to be taken one week before the study and with the exception of the study product no concomitant medication was permitted during the study. Participants were prohibited from alcohol consumption 24 hours prior to the study and smokers were included only if they smoked less than 10 cigarettes per day. All participants were free of skin, cardiorespiratory, and neurological conditions. None of the participants were chronic smokers, and none were taking prescription medications.

The inclusion of human participants in the studies followed the tenets of the Declaration of Helsinki promulgated in 1964 and its amendments. The study protocol was approved by the Rhodes University Ethical Standards Committee (RUESC).

\section{Study design \\ Dose duration study}

The approach described in the FDA's VCA guidance (4) which involves a pilot study with a small number of participants using the reference product to determine the dose duration where the maximum sensitivity can be expected $\left(E D_{50}\right)$ using the $E_{\max }$ model was employed to determine the dose duration for topical MTZ creams. The dose duration study involved a single-phase sequential design conducted on 6 healthy human participants (4 males, 2 females) between the ages of 23 and 30 (mean 26 years). Within this group, 4 participants were Black, 1 was of Indian descent and 1 participant had Mixed Race ancestry. Seven sampling sites $(2 \times 2 \mathrm{~cm})$ were demarcated on the volar aspect of the left forearm using a template (shown in Figure 1). Six sites were used for product application and one site was assigned as a blank. Approximately $15 \mathrm{mg}$ of the reference cream was accurately dispensed from a calibrated dispenser and applied to each application site at time zero. Each site was exposed to the cream for a different dose duration $(1,2,4,6,8$, and $10 \mathrm{~h}$ respectively), after which the residual formulation was removed, and the site was sequentially stripped using 20 preweighed tape strips. The tape strips were weighed again immediately after stripping to determine the amount of SC removed. The designation of the sites was randomised between participants. The tape strips were extracted and analysed using the validated UPLC method. To determine the thickness of the SC on each participant, the blank site also underwent TS and TEWL measurements 
after each strip. The tape strips used on the blank site were spiked with standard solutions to obtain a calibration curve. From these data a profile of extent of drug penetrated through the skin $(A U C)$ vs dose duration was plotted and the $E D_{50}$ determined.

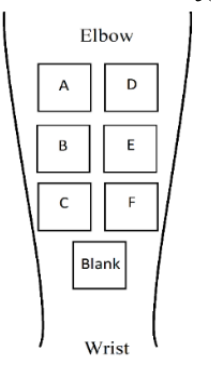

Figure 1. Application template for the dose duration study

\section{Bioequivalence study}

The pivotal study was conducted to demonstrate the ability of the TS method for bioequivalence assessment by comparing the reference product to itself as a positive control using both arms of 10 healthy human participants and also included products with lower $\left(T_{2}\right)$ and higher $\left(T_{3}\right)$ strengths of MTZ to serve as negative controls. In addition, a further cream product $\left(\mathrm{T}_{1}\right)$ containing $0.75 \% \mathrm{MTZ}$ was tested for BE versus the reference product (Metrocreme ${ }^{\circledR}, \quad 0.75 \% \quad$ MTZ). The participants ( 4 males, 6 females) were between the ages of 23 and 31 (mean 27 years) and 6 were Black, 3 were of Indian descent and 1 participant was White. Five sampling sites $(2 \times 2 \mathrm{~cm})$ were demarcated on the volar aspect of each of the forearms using a template (shown in Figure 2). While four sites were used for product application, one site on each arm was assigned as a blank. Approximately $15 \mathrm{mg}$ of the test cream was accurately dispensed and applied to each application site as described above. However, since the duration of application was the same for all the sites, the application was staggered for practicality and convenience. Each site was exposed to the test cream for a time ( $E D_{50}$ from the DD study), after which the residual formulation was removed, and the site was sequentially stripped using 20 tape strips. The designation of the treatment sites was randomised between participants. Each arm of every participant received one treatment each of the reference and the three test products.

\section{Templates}

A double template design was used to prevent contamination due to lateral spreading by preparing an application that was slightly larger than the stripping area in order to minimise inter-site variability (24). The application template was prepared using PVC Binding Covers obtained from a local stationery store. Squares $(2.25 \times 2.25 \mathrm{~cm})$ were cut to expose the application sites. The corners of each square were demarcated using a permanent marker to delineate the application sites after the removal of the template. A sampling template consisting of a single $2 \times 2 \mathrm{~cm}$ square was prepared using Tegaderm ${ }^{\circledR}$ Transparent Film Dressing (3M, St. Paul, MN, USA) since it is a medical tape, hence unlikely to cause any allergic reactions. Following the removal of the product from each site, the sampling template was affixed onto the respective site to facilitate stripping of the specific area on that site.

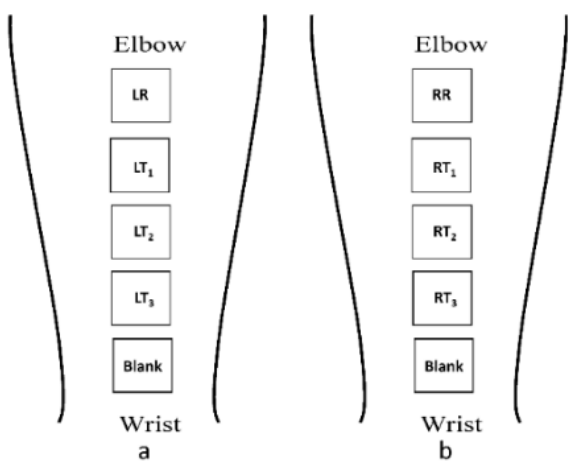

Figure 2. Application Template for BE Phase for (a) Left Arm and (b) Right Arm

\section{Product application}

An Eppendorf dispenser, dial set at 1.5, was used to deliver 1 dose of the formulation, totalling $\sim 15 \mathrm{mg}$ of cream ( $0.11 \mathrm{mg}$ of MTZ) per site. This amount of formulation was in accordance with previous DPK studies (24) as well as the pilot studies carried out as part of TS method development. A pre-weighed fused capillary tube was used to spread the product within the area delineated by the template.

\section{Product removal}

After the required dose duration had elapsed the residual formulation from each site was removed using cotton wool swabs -3 wipes with a swab dipped in lukewarm water and squeezed followed by 2 wipes with a dry swab. Care was taken to remove all the formulation visible.

\section{Tape stripping}

To harvest the SC, each tape strip was placed on the demarcated site and uniform pressure was applied using a roller 10 times in both upward and downward motion. The strip was removed with a single upward pull (shown in Figure 3) and immediately weighed to prevent SC desiccation. 
The direction of stripping was rotated $(\uparrow \mathrm{N}, \rightarrow \mathrm{E}, \downarrow \mathrm{S}$, $\leftarrow \mathrm{W})$ to ensure uniform removal of SC (24).

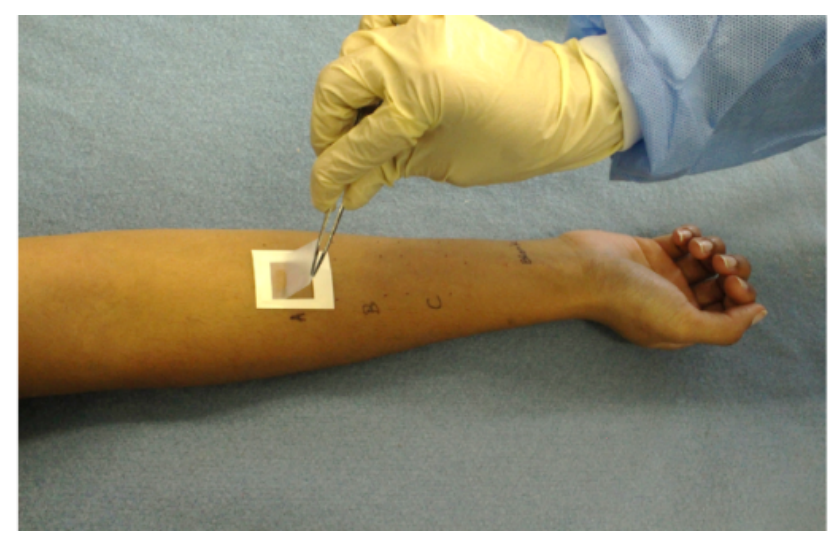

Figure 3. Removal of tape strip with a single upward pull

\section{TEWL measurements}

TEWL measurements were taken at the blank site using a closed chamber Delfin ${ }^{\circledR}$ VapoMeter (Delfin Technologies Ltd., Finland). An initial reading was taken to determine the initial TEWL and then 20 tape strips were used to harvest the SC. After the removal of each tape strip, the VapoMeter was placed vertically and a reading was taken within 10 $\mathrm{s}$ of stripping. The tape strips were weighed immediately after stripping. Once the TEWL reading was completed, the next strip was promptly applied to the site to prevent water loss.

\section{Data Analysis}

\section{Standardisation of skin thickness}

The thickness of the SC varies considerably between individuals, therefore it was necessary to determine the SC thickness for each participant so as to normalise the data obtained. In order to do this, the TEWL readings and TS data collected from the blank site were used and total thickness of the SC determined using Equation 1 (44).

$$
1 / T E W L_{x}=H-x
$$

(Equation 1)

where $T E W L_{x}=$ transepidermal water flux when $x$ mm of skin is removed, $H=$ total thickness of the $\mathrm{SC}$.

Assuming that the SC adhering to each tape strip is uniform and has a density of $1 \mathrm{~g} / \mathrm{cm}^{3}(45), x$ can be calculated from the SC mass. The $x$-intercept of the plot $1 / T E W L_{x} v s x$ provides the value of $H$.

\section{Calculation of AUC}

The amount MTZ per tape strip was plotted against relative SC depth and the area under this curve $(A U C)$ was obtained using the trapezoidal rule. The relative SC depth was calculated by dividing the thickness of SC removed by a particular tape strip $(x)$ by $H(25,39)$. The result was expressed as a percentage.

\section{Dose duration profile}

The dose duration profile was constructed using the mean of the $A U C$ values obtained for each dose duration $(\mathrm{n}=6)$. An $A U C$ value of 0 was assumed at time $=0$ as no MTZ penetration could have occurred. This 6-point profile was fitted to the $E_{\max }$ model and the $E D_{50}$ was calculated using GraphPad Prism Version 4 (GraphPad ${ }^{\mathrm{TM}}$ Software, San Diego, California, USA).

\section{Bioequivalence assessment}

An $A U C_{\text {test }}$ and $A U C_{\text {reference }}$ value was determined for each participant by taking the mean $(\mathrm{n}=2)$ of the $A U C$ values for each test and reference cream. The study was considered to have a paired/ crossover design since each participant received both the reference and test products.

ln-transformed $A U C$ values were evaluated by ANOVA using Proc GLM in SAS ${ }^{\circledR}$ (Statistical Analysis System) version 9.1.3. The statistical model contained terms for subject and treatment effects. The point estimate and the $90 \%$ CI for the test-to-reference ratio were calculated. The results were based on Schuirmann's two one-sided test (TOST) (46) which required that the $90 \% \mathrm{CI}$ of the ratio be contained within the interval $0.80-1.25$ to demonstrate bioequivalence. The residual error term from the ANOVA (MSE) was used to estimate the within-subject CV according to Equation 2.

$$
C V(\%)=\left(e^{M S E}-1\right)^{1 / 2} \times 100
$$

(Equation 2)

The statistical power was calculated using the Microsoft ${ }^{\circledR}$ Excel (version 16.23) spreadsheet accompanying the $5^{\text {th }}$ edition of Pharmaceutical Statistics: Practical and Clinical Applications (47).

For the criss-cross data, Proc GLM procedure of $\mathrm{SAS}^{\circledR}$ was used for statistical analysis (48). The variability between the arms of the subjects was assessed using ANOVA and VARCOMP in SAS ${ }^{\circledR}$ where the results indicated an extremely low degree of variability. 


\section{RESULTS}

\section{UPLC method validation}

The developed UPLC method was validated for the analysis of MTZ following TS studies in human participants in accordance with the ICH guidelines.
Table 1 depicts the validation data in terms of selectivity, linearity, accuracy, precision, robustness and sensitivity as well as sample stability up to 7 days.

Table 1. Predefined acceptance criteria and the results obtained from the UPLC validation for the TS method

\begin{tabular}{|c|c|c|c|c|}
\hline Parameter & Acceptance criteria & \multicolumn{3}{|l|}{ Results } \\
\hline $\begin{array}{l}\text { Selectivity } \\
\text { and } \\
\text { specificity }\end{array}$ & $\begin{array}{l}R T_{p}-R T_{p m}<10 \% \\
T C_{n}=0 \mu \mathrm{g} / \mathrm{mL} \\
T C_{n m}=0 \mu \mathrm{g} / \mathrm{mL}\end{array}$ & \multicolumn{3}{|c|}{$\begin{array}{l}R T_{p}-R T_{p m}=0.09 \% \\
T C_{n}=0 \mu \mathrm{g} / \mathrm{mL}, T C_{n m}=0 \mu \mathrm{g} / \mathrm{mL}\end{array}$} \\
\hline Linearity & $\begin{array}{l}75 \% \text { of the standards meet the } \\
\text { following criteria: } \\
\text { For } 0.1-5 \mu \mathrm{g} / \mathrm{mL}: \\
T C_{\text {meas, }, \text { lin }} \in\left[T C_{\text {nom }} \pm 15 \%\right] \\
\text { For } 0.05 \mu \mathrm{g} / \mathrm{mL}: \\
T C_{\text {meas, }} \text {,in } \\
\left.R^{2} \geq 0.95 C_{\text {nom }} \pm 20 \%\right]\end{array}$ & \multicolumn{3}{|c|}{$\begin{array}{l}30 \text { out of } 30 \text { standards }(100 \%) \text { met the acceptance criteria } \\
R^{2} \geq 0.99\end{array}$} \\
\hline Accuracy & $\begin{array}{l}\text { For } T S_{5} \text { and } T S_{l} \\
T C_{m e a s, a c} \in\left[T C_{n o m} \pm 15 \%\right] \\
\text { For } T S_{0.1} \\
T C_{\text {meas }, a c} \in\left[T C_{\text {nom }} \pm 20 \%\right]\end{array}$ & \multicolumn{3}{|c|}{$\begin{array}{l}\text { For } T S_{5} \text { and } T S_{l} \mathrm{CV} \text { was } 0.46 \text { and } 2.03 \% \\
\text { For } T S_{0.1} \mathrm{CV} \text { was } 4.56 \%\end{array}$} \\
\hline \multirow[t]{2}{*}{ Precision } & $\begin{array}{l}\text { Intra-day CV }<15 \% \text { for } T S_{5} \text { to } T S_{1} \\
\text { and } C V<20 \% \text { for } T S_{0.1}\end{array}$ & \multicolumn{3}{|c|}{$\begin{array}{l}1.5 \%, 0.3 \% \text { and } 0.7 \% \text { for } T S_{5} \\
1.5 \%, 0.7 \% \text { and } 2.0 \% \text { for } T S_{1} \\
1.6 \%, 1.7 \% \text { and } 7.4 \% \text { for } T S_{0.1}\end{array}$} \\
\hline & $\begin{array}{l}\text { Inter-day CV }<15 \% \text { for } T S_{5} \text { to } T S_{1} \\
\text { and } \mathrm{CV}<20 \% \text { for } T S_{0.1}\end{array}$ & \multicolumn{3}{|l|}{$\begin{array}{l}4.8 \% \text { for } T S_{5} \\
2.1 \% \text { for } T S_{I} \\
11.0 \% \text { for } T S_{0.1}\end{array}$} \\
\hline Recovery & Recoveries $>85 \%$ & \multicolumn{3}{|l|}{$\begin{array}{l}93.3 \% \text { for } T S_{5} \\
94.5 \% \text { for } T S_{1} \\
87.5 \% \text { for } T S_{0.1}\end{array}$} \\
\hline \multirow[t]{3}{*}{ Robustness } & \multirow[t]{3}{*}{$\begin{array}{l}\text { Inter-run } \mathrm{CV}<15 \% \text { for } T S_{5} \\
\text { to } T S_{l} \text { and } \mathrm{CV}<20 \% \text { for } T S_{0.1}\end{array}$} & $\begin{array}{l}\text { Lower temper: } \\
1.1 \% \text { for } T S_{5} \\
0.6 \% \text { for } T S_{1} \\
0.1 \% \text { for } T S_{0.1}\end{array}$ & $\mathrm{e}\left(22.5^{\circ} \mathrm{C}\right)$ & $\begin{array}{l}\text { Higher temperature }\left(27.5^{\circ} \mathbf{C}\right) \\
1.4 \% \text { for } T S_{5} \\
0.1 \% \text { for } T S_{1} \\
1.4 \% \text { for } T S_{0.1}\end{array}$ \\
\hline & & $\begin{array}{l}\text { Lower flow rat } \\
7.2 \% \text { for } T S_{5} \\
6.3 \% \text { for } T S_{1} \\
8.3 \% \text { for } T S_{0.1}\end{array}$ & $.18 \mathrm{~mL} / \mathrm{min}$ ) & $\begin{array}{l}\text { Higher flow rate }(\mathbf{0 . 2 2} \mathbf{~} \mathbf{L L} / \mathbf{m i n}) \\
6.6 \% \text { for } T S_{5} \\
6.3 \% \text { for } T S_{l} \\
3.8 \% \text { for } T S_{0.1}\end{array}$ \\
\hline & & \multicolumn{3}{|c|}{$\begin{array}{l}\text { Different column of the same type } \\
11.0 \% \text { for } T S_{5} \\
9.9 \% \text { for } T S_{1} \\
1.4 \% \text { for } T S_{0.1}\end{array}$} \\
\hline $\begin{array}{l}\text { Stability after } \\
7 \text { days }\end{array}$ & $\begin{array}{l}\text { For } T S_{5} \text { and } T S_{1} \\
T C_{m e a s, a c} \in\left[T C_{n o m} \pm 15 \%\right] \\
\text { For } T S_{0.1} \\
T C_{\text {meas }, a c} \in\left[T C_{\text {nom }} \pm 20 \%\right]\end{array}$ & $\begin{array}{l}\text { Bench-top } \\
5.1 \% \text { for } T S_{5} \\
4.1 \% \text { for } T S_{1} \\
4.4 \% \text { for } T S_{0.1}\end{array}$ & $\begin{array}{l}\text { UPLC machine } \\
4.5 \% \text { for } T S_{5} \\
5.4 \% \text { for } T S_{1} \\
2.9 \% \text { for } T S_{0.1}\end{array}$ & $\begin{array}{l}\text { Refrigerator } \\
1.4 \% \text { for } T S_{5} \\
1.7 \% \text { for } T S_{1} \\
1.3 \% \text { for } T S_{0.1}\end{array}$ \\
\hline $\begin{array}{l}\text { LLOQ and } \\
\text { LOD }\end{array}$ & To be reported & LLOQ: $0.05 \mu q$ & & LOD: $0.02 \mu \mathrm{g} / \mathrm{mL}$ \\
\hline
\end{tabular}




\section{Dose duration study}

The profile of $1 / T E W L v s$ cumulative skin depth obtained from the blank sites showed good linear correlation, with $R^{2}$ values ranging from 0.89 to 0.97 in accordance with previously published reports (15). The mean SC thickness of the 6 participants was found to be $10.6 \pm 1.2 \mu \mathrm{m}$ which was within the expected range. The mean fraction of SC harvested from the sites after 20 strips was $72.6 \pm 8.2 \%$. The data showed that MTZ penetration was rapid up to $6 \mathrm{~h}$, after which it appeared to level out indicating that steady state had been achieved (shown in Figure 4). Since the $E D_{50}$ obtained was $60.9 \mathrm{~min}$, a more practical and convenient dose duration of $60 \mathrm{~min}$ (i.e. $1 \mathrm{~h}$ ) was chosen for the subsequent studies.

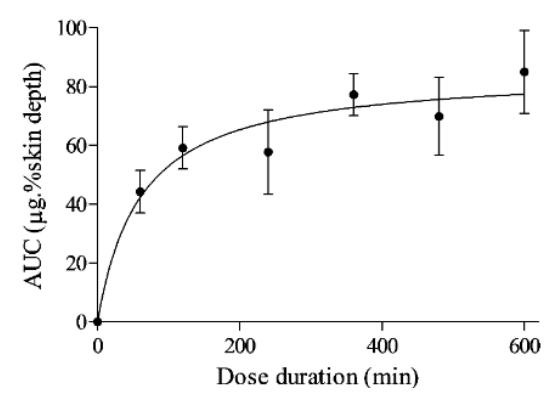

Figure 4. Fitted dose- response curve generated $\left(E_{\max }=85.3 \mu \mathrm{g} . \%\right.$ skin depth and $\left.E D_{50}=60.9 \mathrm{~min}\right)$

\section{Bioequivalence study}

The SC thickness between arms of each subject did not vary greatly between the two arms $(\mathrm{CV}<$ $10 \%$ ) (shown in Figure 5). Almost all the SC was consistently harvested in all the participants and the mean fraction of SC removed was $90.4 \pm$ $7.0 \%(\mathrm{n}=100)$ with a $\mathrm{CV}=7.8 \%$. The mean thickness of SC between participants was found to be $8.2 \pm 1.6 \mu \mathrm{m}$.

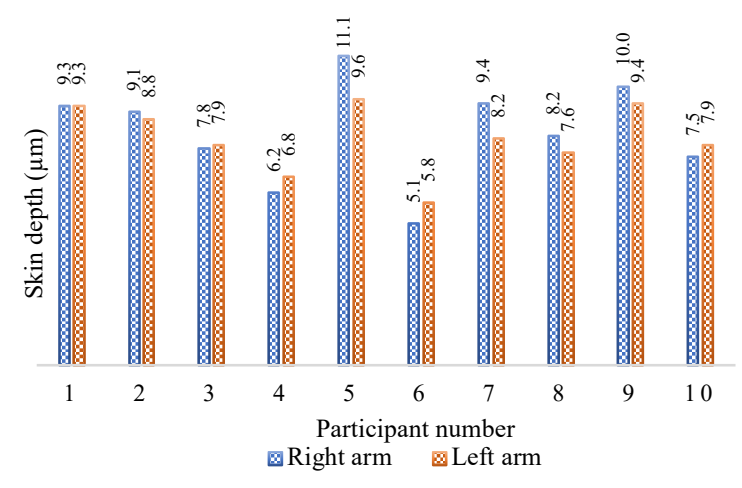

Figure 5. Comparison of SC thickness between arms of the participants
When the reference product was compared against itself, the $90 \%$ CI limits for $A U C_{\text {test }} / A U C_{\text {reference }}$ ratio was found to be well within the $0.80-1.25$ limits confirming BE. According to the results, 6 participants were sufficient to demonstrate BE with an $80 \%$ power (Table 2).

Table 2. Summary of results for the BE confirmation study

\begin{tabular}{ll}
\hline \multicolumn{2}{l}{ Reference (left arm) $v$ s Reference (right arm) } \\
\hline $\mathrm{n}$ & 10 \\
\hline$A U C_{\text {left }} / A U C_{\text {right }}$ & 1.05 \\
\hline $\mathrm{CI} 90 \%$ limits & $0.98-1.13$ \\
\hline $\mathrm{CV}(\%)$ & 8.61 \\
\hline Bioequivalent? $(0.80-1.25)$ & Yes \\
\hline $\mathrm{n}$ required for $>80 \%$ power & 6 \\
\hline
\end{tabular}

It was observed that the CI $90 \%$ limits for products $\mathrm{T}_{2}$ and $\mathrm{T}_{3}$ containing $25 \%$ less and $26 \%$ more MTZ than the reference product, respectively when compared with the reference product, Metrocreme ${ }^{\mathbb{B}}$ did not meet $\mathrm{BE}$ acceptance criteria of $0.80-1.25$ as depicted in Table 3 . The $25 \%$ less product's lower $90 \%$ CI limit was below 0.80 and the upper $90 \%$ CI limit for the $26 \%$ higher product was above 1.25 .

Table 3. Summary of results for the BE assessment of $\mathrm{T}_{2}$ and $\mathrm{T}_{3}$ against the reference product

\begin{tabular}{lll}
\hline & $\begin{array}{l}\mathbf{T}_{\mathbf{2}} \text { vs } \\
\text { Reference }^{1}\end{array}$ & $\begin{array}{l}\mathbf{T}_{\mathbf{3}} \text { vs } \\
\text { Reference }^{2}\end{array}$ \\
\hline $\mathrm{n}$ & 10 & 10 \\
\hline AUC $_{\text {test }} /$ $^{2} C_{\text {reference }}$ & 0.79 & 1.25 \\
\hline $\mathrm{CI} 90 \%$ limits & $0.74-0.84$ & $1.18-1.33$ \\
\hline $\mathrm{CV} \%$ & 7.75 & 7.09 \\
\hline $\begin{array}{l}\text { Bioequivalent? } \\
(0.80-1.25)\end{array}$ & No & No \\
\hline 'post-hoc power $=0.01, \mathrm{p} \leq 0.05,{ }^{2}$ post-hoc power $=0.03, \mathrm{p} \leq 0.05$. \\
\hline
\end{tabular}

A further MTZ formulation, test cream $\left(\mathrm{T}_{1}\right)$ which contained $0.75 \%$ MTZ was compared against the reference product, Metrocreme ${ }^{\circledR}$ $(0.75 \% \mathrm{MTZ})$ for BE assessment. This was done in two ways - (i) the site that received $\mathrm{T}_{1}$ on the right arm was compared to the reference on the left arm of the same participant and vice versa, and (ii) means of the $A U C s$ for each product were calculated using the respective sites on both arms to compare $T_{1}$ and the reference product. Table 4 depicts that the number of participants required for a statistical power of greater than $80 \%$ was less than 10 . The results of the criss-cross analysis indicated that there was no statistically significant 
difference in the results between arms $(\mathrm{p}>0.05)$. The $90 \%$ CI limits were within the range of 0.80 -1.25 thereby establishing BE between the test product, $\mathrm{T}_{1}$ and the reference (Table 4 ) in both cases.

Table 4. Summary of results for the BE assessment of $\mathrm{T}_{1}$ against the reference product

\begin{tabular}{|c|c|c|}
\hline & $\begin{array}{l}T_{1} v s \text { Reference } \\
\text { (criss-cross) }\end{array}$ & $\begin{array}{l}\mathrm{T}_{1} \text { vs } \\
\text { Reference }^{1}\end{array}$ \\
\hline $\mathrm{n}$ & 10 & 10 \\
\hline$A U C_{\text {test }} / A U C_{\text {reference }}$ & 1.00 & 1.00 \\
\hline CI $90 \%$ limits & $0.95-1.05$ & $0.95-1.06$ \\
\hline $\mathrm{CV}$ & 9.84 & 7.07 \\
\hline $\begin{array}{l}\text { Bioequivalent? } \\
(0.80-1.25)\end{array}$ & Yes & Yes \\
\hline $\begin{array}{l}n \text { required for } \\
>80 \% \text { power }\end{array}$ & 7 & 5 \\
\hline
\end{tabular}

In addition, the variability estimates between subjects, arms and formulations are summarised in Table 5.

Table 5. Variability between subjects, arms and products for $T_{1}$ against the reference product

\begin{tabular}{lclll}
\hline Parameter & Subjects & Arms & Products & Error $^{1}$ \\
\hline $\begin{array}{l}\text { Variability } \\
\text { Estimates }\end{array}$ & 15.9 & 3.2 & 18.8 & 11.1 \\
$\begin{array}{l}\text { (CV\%) } \\
{ }^{1} \text { Residual } \\
(\mathrm{CV} \%))\end{array}$ & & & & \\
\hline
\end{tabular}

\section{DISCUSSION}

The UPLC method met the acceptance criteria for all the validation parameters and was found to be selective, linear over a range of $0.05-5 \mu \mathrm{g} / \mathrm{mL}$, accurate, precise, robust, sensitive with an LLOQ of $0.05 \mu \mathrm{g} / \mathrm{mL}$ and offering sample stability up to 7 days.

Importantly, it was seen that the duration of contact with the formulation or the position of the site on the arm did not influence the percentage of SC removed. The exponential increase in the penetration of MTZ observed was in accordance with the study conducted by de Araujo et al. (33), which indicated uptake up to $6 \mathrm{~h}$ application. Measurements were continued for various times at sites after removal of the dose at $6 \mathrm{~h}$ where the amount of MTZ decreased which the authors describe as the clearance phase. Generally PK measures of maximum drug quantity per unit area $\left(\mathrm{ng} / \mathrm{cm}^{2}\right)$ in the SC $\left(Q_{\max }\right)$, and the area under the curve $(A U C)$ deduced from the DPK profile are not the same as the PK parameters, peak plasma drug concentration $\left(C_{\max }\right)$ and $A U C$, respectively, evaluated after oral administration of a systemically absorbed drug. With the oral route of administration where these parameters are affected by the kinetics of drug absorption, distribution, metabolism and elimination, $C_{\max }$ is measured as a concentration (drug per unit of blood volume) unlike $Q_{\max }$ in topical delivery which, importantly, is expressed as an amount. Furthermore, the mechanisms of uptake into and clearance from the $\mathrm{SC}$ are primarily controlled by penetration across the skin, usually the SC itself. $Q_{\max }$, therefore, represents the amount that reaches the SC. The PK parameter, clearance, therefore can only be attributed to the manual removal of residual amounts on the skin by wiping the sites and thus the concept of a volume of distribution does not apply, rendering $A U C$ in $\mathrm{SC}$ to be different from the systemic $A U C$ obtained from a plasma drug level-time profile. These technicalities led to the development of the Relative Depth method $(22,39,41)$ which is different from the FDA proposed method where the DPK profile is represented by the total amount in SC $\left(\mathrm{ng} / \mathrm{cm}^{2}\right)$ as a function of time (h). Although this approach involves the measurement of drug amount at only one DD in the uptake phase instead of 8 as in the FDA approach, it necessitates the quantification of the SC removed in each layer (ng) and derivation of the total SC thickness $(\mathrm{cm})$ from the removed mass with the help of assumptions. Due to the incorporation of numerous assumptions to estimate these parameters, and the complicated calculations involved, the credibility of the method to directly reflect the rate and extent of "absorption" or input into the skin has been contested. Furthermore, the movement of drug across the SC layer throughout the tape stripping procedure is not taken into consideration which may result in inconsistent amounts at that location when the exposure ends unless tape stripping is rapidly conducted ultimately affecting the accuracy in the estimation of thermodynamic parameters such as SC-vehicle partition coefficient of the drug, and the diffusivity of the drug across the SC of thickness (49). Therefore, a study design in accordance with the withdrawn FDA DPK draft guidance (15) does not seem to be relevant for topical formulations. After topical application of a drug, the amount found at the site of action is determined primarily by SC 
penetration and processes such as partitioning, diffusion and keratin binding. This is quite different for drugs which are intended to be absorbed into the systemic circulation where the drug plasma concentration $v s$ time profile is controlled by absorption, distribution, metabolism and excretion. Furthermore, since the objective of this study was to identify a dose duration with adequate sensitivity to distinguish between different products containing MTZ to establish BE, the inclusion of clearance phases was deemed irrelevant.

Since the SC thickness and fraction of SC removed were essentially the same in an individual participant, the sites treated with the same product on both arms were considered to be comparable. Hence, to demonstrate the utility of the developed method to confirm $\mathrm{BE}$, the reference product on the left arms of the participants was compared with that on their right arms. No significant difference was observed between the arms $(p>0.05)$ where the 90\% confidence interval for the arm-to-arm ratio met bioequivalence criteria.

The ability of the method to demonstrate BE was confirmed from the results comparing the reference product to itself, whereas the discriminatory power of the method was shown by the products used as negative controls accordingly failed to be shown as bioequivalent to the reference product since the probability of $\mathrm{BE}$ was less than $5 \%$ as shown in Table 3. Another cream formulation containing $0.75 \%$ MTZ $\left(\mathrm{T}_{1}\right)$ when compared with the reference product was shown to be bioequivalent.

The CV\% between subjects which is a function of the difference between the subjects indicated only moderate subject-to-subject differences, was well within the range seen for a standard crossover BE study based on blood concentration results. The $\mathrm{CV} \%$ between arms were very low indicating that results obtained for the right arm are consistent with those obtained for the left arm. The residual error $\mathrm{CV} \%$ was relatively low for an in-vivo $\mathrm{BE}$ method, comparable to what is seen in a typical crossover blood level BE study involving a low variability drug demonstrating that the methodology had excellent precision.

\section{CONCLUSION}

A TS method for the assessment of the penetration of topical cream products containing
MTZ into the SC of human participants was successfully developed and optimised by taking into consideration the variables that could affect the results. Furthermore, the method was applied to determine the duration of application of the formulation necessary to provide adequate sensitivity to discriminate possible significant differences between creams containing MTZ should this occur. The dose duration was subsequently used to establish bioequivalence between creams containing MTZ. The inclusion of positive and negative controls further confirmed that the method had the necessary discriminatory power to distinguish between bioequivalent products and products that were truly not bioequivalent. In addition, this TS method involved the use of both forearms of each participant which resulted in an important advantage of significantly reducing the number of human subjects required to show BE whilst still retaining a statistical power $>80 \%$. The data also provided compelling evidence that the developed TS method has the potential to be a cost-effective surrogate alternative for lengthy and expensive clinical trials thereby facilitating faster development of generic products which would, in turn, lower the economic burden of healthcare.

\section{ACKNOWLEDGEMENT}

GALENpharma GmbH, Kiel, Germany, is gratefully acknowledged for providing the test creams.

\section{CONFLICT OF INTEREST STATEMENT}

This study was financially supported by the Biopharmaceutics Research Institute (BRI), Rhodes University, South Africa and was a part of the $\mathrm{PhD}$ research carried out by Seeprarani Rath under the supervision of Isadore Kanfer. Ashmita Ramanah provided technical and laboratory assistance during the study and Charles Bon helped with the statistical analysis. The authors declare that they have no conflict of interest related to this work.

\section{REFERENCES}

1. Generic Drug Access \& Savings Report. Washington: Association for Accessible Medicines. 2018. Available from: https://accessiblemeds.org/search?search $=2018+\mathrm{g}$ eneric + drug + access + and + savings + report. [cited 
20 May 2020]

2. Li DG, Joyce C, Mostaghimi A. Association between Market Competition and Prices of Generic Topical Dermatology Drugs. JAMA Dermatology. 2018;154(12):1441-6. DOI: 10.1016/j.ijpharm.2018.02.032.

3. Preet P, Kaur A. Challenges in bioequivalence assessment of topical dermatological dosage forms. Res J Life Sci Bioinformatics, Pharmaceutical Chem Sci. 2018;4(1):21-8. DOI: $10.26479 / 2017.0401 .02$.

4. US Food and Drug Administration, Center for Drug Evaluation and Research. Guidance for Industry: Topical Dermatologic Corticosteroids In vivo Bioequivalence. Rockville (MD): US Food and Drug Administration. 1995. Available from: https://www.fda.gov/media/70931/download. [cited 20 October 2020]

5. US Food and Drug Administration, Office of Generic Drugs. Draft Guidance on Acyclovir: Ointment. Recommended Mar 2012; Revised Feb 2019, Sept 2019. Available from: https://www.accessdata.fda.gov/drugsatfda docs/ psg/PSG 018604.pdf. [cited 20 October 2020]

6. US Food and Drug Administration, Office of Generic Drugs. Draft Guidance on Acyclovir: Cream. Recommended Dec 2014; Revised Dec 2016. Available from: https:/www.accessdata.fda.gov/drugsatfda docs/ psg/Acyclovir topical\%20cream RLD\%2021478 RV12-16.pdf. [cited 20 October 2020]

7. US Food and Drug Administration, Office of Generic Drugs. Draft Guidance on Dapsone. Recommended Dec 2014; Revised Oct 2017, Nov 2018, Nov 2019. Available from: https://www.accessdata.fda.gov/drugsatfda docs/ psg/PSG 021794.pdf. [cited 20 October 2020]

8. US Food and Drug Administration, Office of Generic Drugs. Draft Guidance on Docosanol. Recommended Oct 2017. Available from: https://www.accessdata.fda.gov/drugsatfda docs/ psg/Docosanol topical\%20cream NDA\%200209 41 RC08-17.pdf. [cited 20 October 2020]

9. US Food and Drug Administration, Office of Generic Drugs. Draft Guidance on Ivermectin: Cream. Recommended Dec 2016. Available from: https://www.accessdata.fda.gov/drugsatfda docs/ psg/Ivermectin $\% 20$ Topical $\% 20$ Cream $\% 201 \% 20$ NDA $\% 20206255 \% 20 P S G \% 20$ Page $\% 20 R V \% 20$ May\%202019.pdf. [cited 22 October 2020]

10. US Food and Drug Administration, Office of Generic Drugs. Draft Guidance on Cyclosporin. Recommended Oct 2017; Revised Sept 2018, May 2019. Available from: https:/www.accessdata.fda.gov/drugsatfda docs/ psg/Cyclosporine ophthalmic\%20emulsion RLD $\% 20050790$ RV09-16.pdf. [cited 22 October 2020]
11. Higo N, Naik A, Bommannan DB, Potts RO, Guy RH. Validation of reflectance infrared spectroscopy as a quantitative method to measure percutaneous absorption in vivo. Pharm Res. 1993;10(10):1500-6. DOI: 10.1023/a:1018987612155.

12. Borsadia S, Ghanem A, Seta Y, Higuchi WI, Flynn GL, Behl CR, et al. Factors to be considered in the evaluation of bioavailability and bioequivalence of topical formulations. Skin Pharmacol. 1992;5(3):129-45. DOI: 10.1159/000211030.

13. Wagner H, Kostka KH, Lehr CM, Schaefer UF. Interrelation of permeation and penetration parameters obtained from in vitro experiments with human skin and skin equivalents. J Control Release. $2001 \quad$ Aug;75(3):283-95. DOI: 10.1016/s0168-3659(01)00396-0.

14. Marks R, Dykes P. Plasma and cutaneous drug levels after topical application of piroxicam gel: a study in healthy volunteers. Skin Pharmacol. 1994;7(6):340-4. DOI: 10.1159/000211316.

15. US Food and Drug Administration, Center for Drug Evaluation and Research. Guidance for Industry: Topical Dermatological Drug Product NDAs and ANDAs - In Vivo Bioavailability, Bioequivalence, In Vitro Release, and Associated Studies. Rockville (MD): US Food and Drug Administration. 1998. Available from: https://pdfs.semanticscholar.org/18c4/a3a9696003 e353f2cc7830560e828a89eed5.pdf? ga=2.20407 352.1800331445 .1590846625 394870020.1590846625. [cited 15 October 2020]

16. Clausen M-L, Slotved H-C, Krogfelt KA, Agner T. Tape Stripping Technique for Stratum Corneum Protein Analysis. Sci Rep. 2016;6:19918. DOI: $10.1038 /$ srep19918.

17. US Department of Health and Human Services, US Food and Drug Administration. Guidance for Industry on Special Protocol Assessment; Availability. Federal Register; 2002 p. 31-2.

18. N'Dri-Stempfer B, Navidi WC, Guy RH, Bunge AL. Improved bioequivalence assessment of topical dermatological drug products using dermatopharmacokinetics. Pharm Res. 2009;26(2):316-28. DOI: 10.1007/s11095-0089742-9.

19. Lu M, Xing H, Chen X, Xian L, Jiang J, Yang T, et al. Advance in bioequivalence assessment of topical dermatological products. Asian J Pharm Sci. 2016;11(6):700-7. DOI: 10.1016/j.ajps.2016.04.008.

20. N'Dri-Stempfer B, Navidi WC, Guy RH, Bunge AL. Optimizing metrics for the assessment of bioequivalence between topical drug products. Pharm Res. 2008;25(7):1621-30. DOI: $10.1007 / \mathrm{s} 11095-008-9577-4$.

21. Pershing LK, Nelson JL, Corlett JL, Shrivastava SP, Hare DB, Shah VP. Assessment of dermatopharmacokinetic approach in the 
bioequivalence determination of topical tretinoin gel products. J Am Acad Dermatol. 2003;48(5):740-51. DOI: 10.1067/mjd.2003.175.

22. Herkenne C, Alberti I, Naik A, Kalia YN, Mathy $\mathrm{FX}$, Préat $\mathrm{V}$, et al. In vivo methods for the assessment of topical drug bioavailability. Pharm Res. 2008;25(1):87-103. DOI: 10.1007/s11095007-9429-7.

23. Nallagundla S, Patnala S, Kanfer I. Application of an Optimized Tape Stripping Method for the Bioequivalence Assessment of Topical Acyclovir Creams. AAPS PharmSciTech. 2018;19(4):156773. DOI: 10.1208/s12249-018-0971-X.

24. Parfitt NR, Skinner M, Bon C, Kanfer I. Bioequivalence of topical clotrimazole formulations: An improved tape stripping method. J Pharm Pharm Sci. 2011;14(3):347-57. DOI: $10.18433 / \mathrm{j} 3 \mathrm{rp} 5 \mathrm{k}$.

25. Herkenne C, Naik A, Kalia YN, Hadgraft J, Guy $\mathrm{RH}$. Ibuprofen transport into and through skin from topical formulations: In vitro-in vivo comparison. J Invest Dermatol. 2007;127:135-42. DOI: $10.1038 /$ sj.jid.5700491.

26. National Institute of Health Sciences. Guideline for Bioequivalence Studies of Generic Products for Topical Use. Setagaya Yoga: National Institute of Health Sciences. 2003. Available from: http://www.nihs.go.jp/drug/beguide(e)/Topical BE-E.pdf. [cited 15 May 2020]

27. Department of Health (Republic of South Africa), Medicines Control Council. Biostudies: Registration of Medicines, Version 6. Pretoria: Medicines Control Council. 2015. Available from: http://www.rrfa.co.za/wpcontent/uploads/2012/11/Biostudies-Jun-15-v6showing-changes.pdf. [cited 15 May 2020]

28. Soares KCC, Santos GML, Gelfuso GM, Gratieri T. An Update of the Brazilian Regulatory Bioequivalence Recommendations for Approval of Generic Topical Dermatological Drug Products. AAPS J. 2015;17(6):1517-8. DOI: 10.1208/s12248-015-9801-1.

29. European Medicines Agency, Committee for Medicinal Products for Human Use. Draft guideline on quality and equivalence of topical products. London: European Medicines Agency; 2018. Available from: https://www.ema.europa.eu/en/documents/scientif ic-guideline/draft-guideline-quality-equivalencetopical-products en.pdf. [cited 15 May 2020]

30. US Food and Drug Administration, Center for Drug Evaluation and Research. Guidance for Industry: Competitive Generic Therapies. Silver Spring (MD): US Food and Drug Administration. 2020. Available from: https://www.fda.gov/media/136063/download. [cited 15 May 2020]

31. Benfeldt E, Hansen SH, Vølund A, Menné T, Shah VP. Bioequivalence of topical formulations in humans: evaluation by dermal microdialysis sampling and the dermatopharmacokinetic method. J Invest Dermatol. 2007;127(1):170-8. DOI: $10.1038 /$ sj.jid.5700495.

32. Navidi W, Hutchinson A, N'Dri-Stempfer B, Bunge A. Determining bioequivalence of topical dermatological drug products by tape-stripping. J Pharmacokinet Pharmacodyn. 2008 Jun;35(3):337-48. DOI: 10.1007/s10928-0089091-7.

33. de Araujo TP, Fittipaldi IM, Bedor DCG, Duarte ML, Cordery SF, Guy RH, et al., et al. Topical bio(in)equivalence of metronidazole formulations in vivo. Int J Pharm. 2018;541(1-2):167-72. DOI: 10.1016/j.ijpharm.2018.02.032.

34. García Ortiz P, Hansen SH, Shah VP, Sonne J, Benfeldt E,. Are Marketed Topical Metronidazole Creams Bioequivalent? Evaluation by in vivo Microdialysis Sampling and Tape Stripping Methodology. Skin Pharmacol Physiol. 2011;24(1):44-53. DOI: 10.1159/000320151.

35. Incecayir T, Agabeyoglu I, Derici U, Sindel S. Assessment of topical bioequivalence using dermal microdialysis and tape stripping methods. Pharm Res. 2011;28(9):2165-75. DOI: 10.1007/s11095-011-0444-3.

36. Cordery SF, Pensado A, Chiu WS, Shehab MZ, Bunge AL, Delgado-Charro MB, et al. Topical bioavailability of diclofenac from locally-acting, dermatological formulations. Int $\mathrm{J}$ Pharm. 2017;529(1-2):55-64.

10.1016/j.ijpharm.2017.06.063.

37. Lodén M, Åkerström U, Lindahl K, Berne B. Bioequivalence determination of topical ketoprofen using a dermatopharmacokinetic approach and excised skin penetration. Int J Pharm. 2004;284(1-2):23-30.

38. Au WL, Skinner M, Kanfer I. Comparison of Tape Stripping with the Human Skin Blanching Assay for the Bioequivalence Assessment of Topical Clobetasol Propionate Formulations. J Pharm Pharm Sci. 2010;13(1):11-20. DOI: $10.18433 / \mathrm{j} 3 \mathrm{c} 01 \mathrm{r}$.

39. Herkenne C, Naik A, Kalia YN, Hadgraft J, Guy RH. Dermatopharmacokinetic prediction of topical drug bioavailability in vivo. J Invest Dermatol. 2007;127:887-94. DOI: 10.1038/sj.jid.5700642.

40. Pirot F, Kalia YN, Stinchcomb AL, Keating G, Bunge A, Guy RH. Characterization of the permeability barrier of human skin in vivo. Proc Natl Acad Sci U S A. 1997;94(4):1562-7. DOI: 10.1073/pnas.94.4.1562.

41. Alberti I, Kalia YN, Naik A, Guy RH. Assessment and prediction of the cutaneous bioavailability of topical Terbinafine, in vivo, in man. Pharm Res. 2001;18(10):1472-5. 10.1023/a:1012217209228.

42. Ozdin D, Kanfer I, Ducharme MP. Novel Approach for the Bioequivalence Assessment of 
Topical Cream Formulations: Model-Based Analysis of Tape Stripping Data Correctly Concludes BE and BIE. Pharm Res. 2020;37(2). DOI: 10.1007/s11095-019-2724-2.

43. International Conference on the Harmonization of technical requirements for the registration of pharmaceuticals for human use, ICH Expert Working Group. ICH harmonized tripartite guideline, Validation of Analytical Procedures: Text and Methodology Q2 (R1), Step 4 version. Geneva: International Conference on the Harmonization of technical requirements for the registration of pharmaceuticals for human use. 2005. Available from: https://database.ich.org/sites/default/files/Q2 R1 Guideline.pdf. [cited 15 May 2020]

44. Kalia YN, Alberti I, Sekkat N, Curdy C, Naik A, Guy RH. Normalization of stratum corneum barrier function and transepidermal water loss in vivo. Pharm Res. 2000;17(9):1148-50. DOI: 10.1023/a:1026474200575.

45. Anderson RL, Cassidy JM. Variations in Physical Dimensions and Chemical Composition of Human
Stratum Corneum. J Invest Dermatol. 1973 Jul;61(1):30-2. DOI: $\quad 10.1111 / 1523$ 1747.ep12674117.

46. Schuirmann DJ. A comparison of the Two OneSided Tests Procedure and the Power Approach for assessing the equivalence of average bioavailability. J Pharmacokinet Biopharm. 1987;15(6):657-80. DOI: 10.1007/BF01068419.

47. Bolton S, Bon C. Pharmaceutical Statistics Practical and Clinical Applications. 4th ed. New York: Marcel Dekker, Inc.; 2004.

48. US Food and Drug Administration, Center for Drug Evaluation and Research. Guidance for industry: Statistical approaches to establishing bioequivalence. Rockville (MD): US Food and Drug Administration. 2001. Available from: https://www.fda.gov/media/70958/download. [cited 15 May 2020]

49. Reddy MB, Stinchcomb AL, Guy RH, Bunge AL. Determining dermal absorption parameters in vivo from tape strip data. Pharm Res. 2002;19(3):2928. DOI: $10.1023 / \mathrm{a}: 1014443001802$. 